\title{
Lepromatous Leprosy: A Commonly Misdiagnosed Disease
}

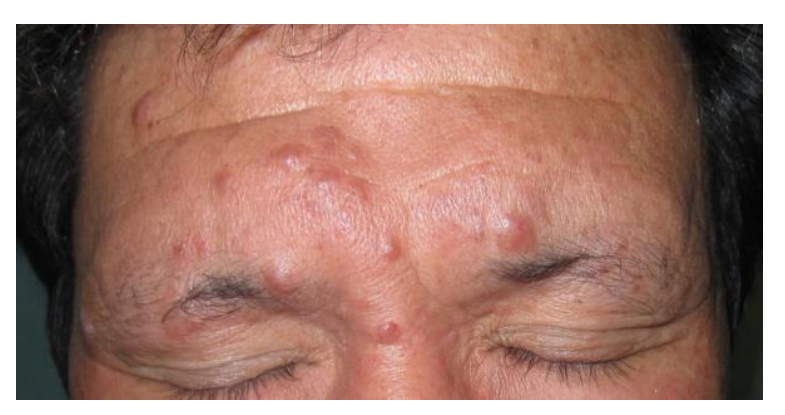

$\mathbf{A}$

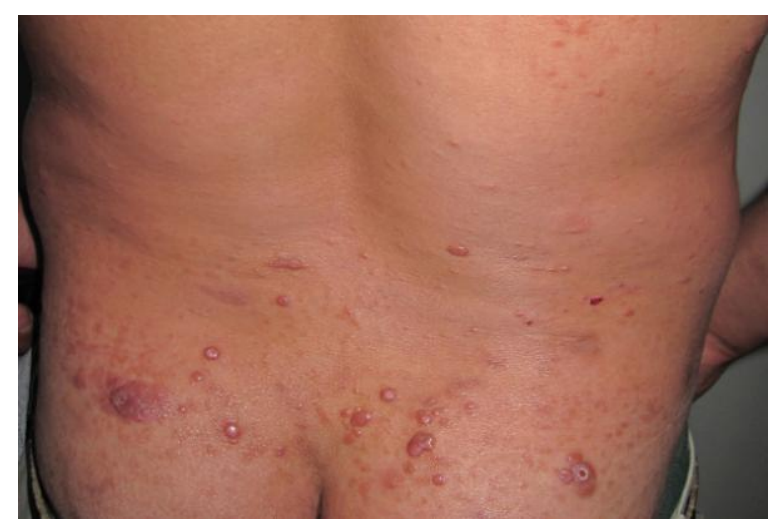

C

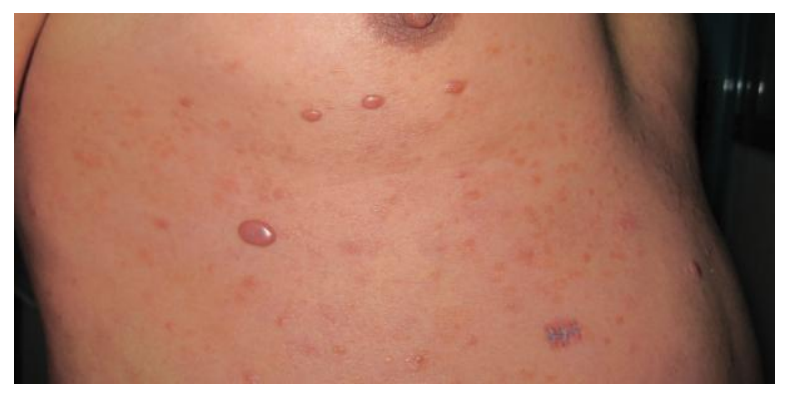

B

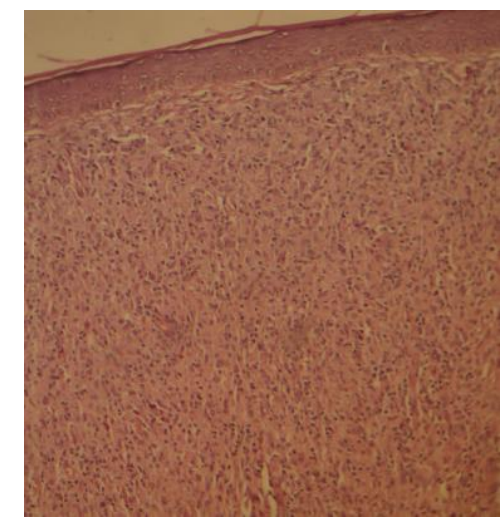

D

FIGURE 1. Erythematous/coppery papules and nodules appearing on the face and body (A-C), with no apparent granuloma (D).

Reza Yaghoobi ${ }^{1}$, Amir Feily ${ }^{1, \star}$, Nastaran Ranjbari ${ }^{2}$, Jalal Lotfi ${ }^{1}$, and Elena Yaghoobi

${ }^{1}$ Department of Dermatology and ${ }^{2}$ Department of Pathology, Jundishapur University of Medical Sciences, Ahvaz, Iran

E-mail: Yaghoobi rz@yahoo.com; dr.feily@yahoo.com; saminjan@yahoo.com; sjlotfi@yahoo.com

Received September 4, 2010; Revised October 4, 2010; Accepted October 6, 2010; Published December 14, 2010

KEYWORDS: lepromatous leprosy, endemic disease 
A 42-year-old man was referred to our department for treatment with an 8-year history of cutaneous nodules of the face, back, trunk, and buttocks. Physical examination was notable for multiple painless erythematous/coppery papules and nodules with loss of the lateral eyebrows (Fig. 1A-C). There was no variation in the cutaneous sensations of temperature, touch, and pain. The patient came from Afghanistan, an area in which lepromatous leprosy is endemic. Histopathologic examination showed infiltration of foamy histocytes and macrophages extending to the subcutaneous fat separated from the epidermis by the narrow granz zone. Apparently there was no granuloma formation (Fig. 1D). In a mucosal smear from his nose and acid fast staining, numerous acid-fast bacilli were seen and a diagnosis of lepromatous leprosy was made. Cutaneous clinical lesions of lepromatous leprosy include cutaneous/subcutaneous firm, translucent, erythematous/coppery, and shiny papules and nodules appearing over an apparently normal skin[1]. Ocular complications are found in $73 \%$ of the patients and loss of eyebrows is the most frequent occurrence[2]. A number of skin diseases may be confused with leprosy. The differential diagnosis of the infiltrated plaque or nodules includes leishmaniasis, syphilis, sarcoidosis, lymphoma, and cutaneous tuberculosis[3]. If any physician sees a patient from an area where this infection is endemic (Asia, subSaharan Africa, South and Central America, the Pacific Islands, and the Philippines)[4] and who has unexplained skin lesions, it is important to consider leprosy. This patient was treated successfully with World Health Organization (WHO)-recommended multibacillary multidrug therapy (MBMDT). The skin lesions resolved with minimal scarring.

\section{REFERENCES}

1. Sehgal, V.N., Aggarwal, A., Srivastava, G., Sharma, N., and Sharma, S. (2005) Evolution of histoid leprosy (de novo) in lepromatous (multibacillary) leprosy. Int. J. Dermatol. 44(7), 576-578.

2. Khan, T., Awan, A.A., Kazmi, H.S., Shah, A.A., Muhammad, S., and Muhammad, S. (2002) Frequency of ocular complications of leprosy in institutionalized patients in NWFP Pakistan. J. Ayub Med. Coll. Abbottabad 14(4), $29-33$.

3. Sehgal, V.N. (1994) Leprosy. Dermatol. Clin. 12(4), 629-644.

4. Thompson, A.M., Lynn, A.A., Robson, K., Joyce, M.P., Fivenson, D.P., and Scollard, D. (2003) Lepromatous phlebitis of the external jugular vein. J. Am. Acad. Dermatol. 49(6), 1180-1182.

This article should be cited as follows:

Yaghoobi, R., Feily, A., Ranjbari, N., Lotfi, J., and Yaghoobi, E. (2010) Lepromatous leprosy: a commonly misdiagnosed disease. TheScientificWorldJOURNAL 10, 2348-2349. DOI 10.1100/tsw.2010.206. 


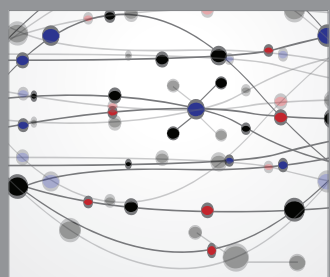

The Scientific World Journal
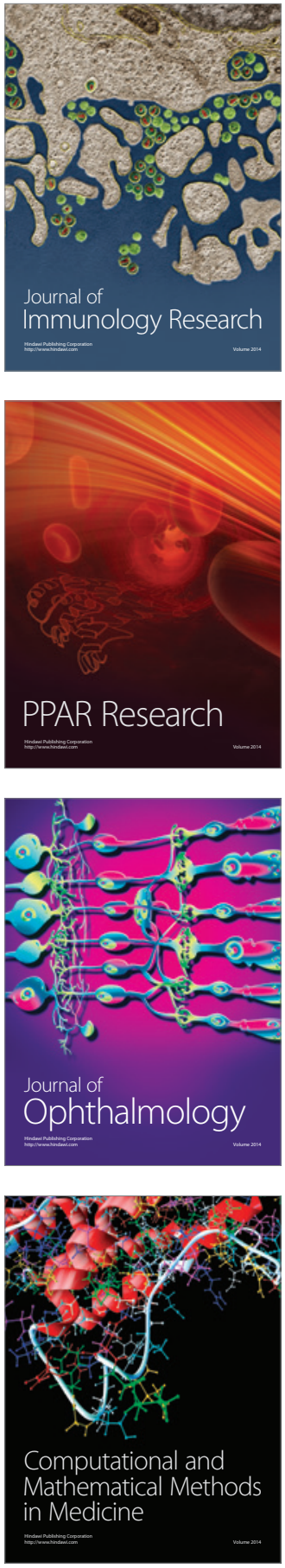

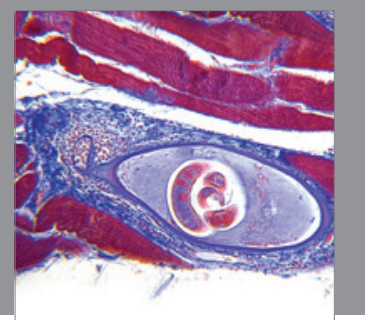

Gastroenterology

Research and Practice
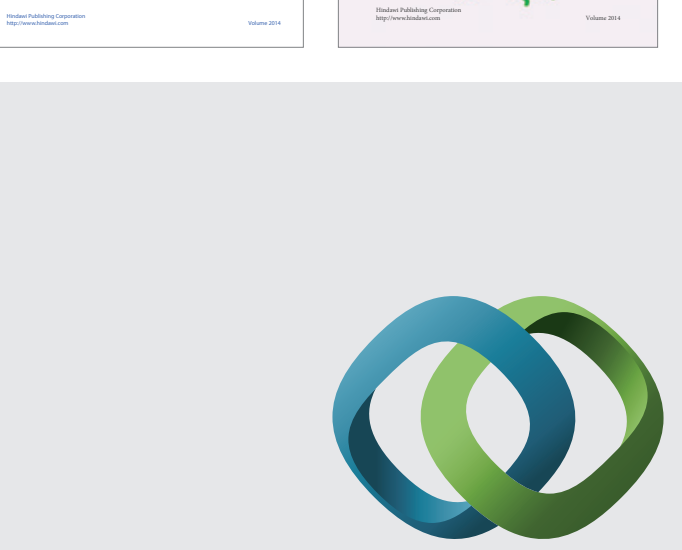

\section{Hindawi}

Submit your manuscripts at

http://www.hindawi.com
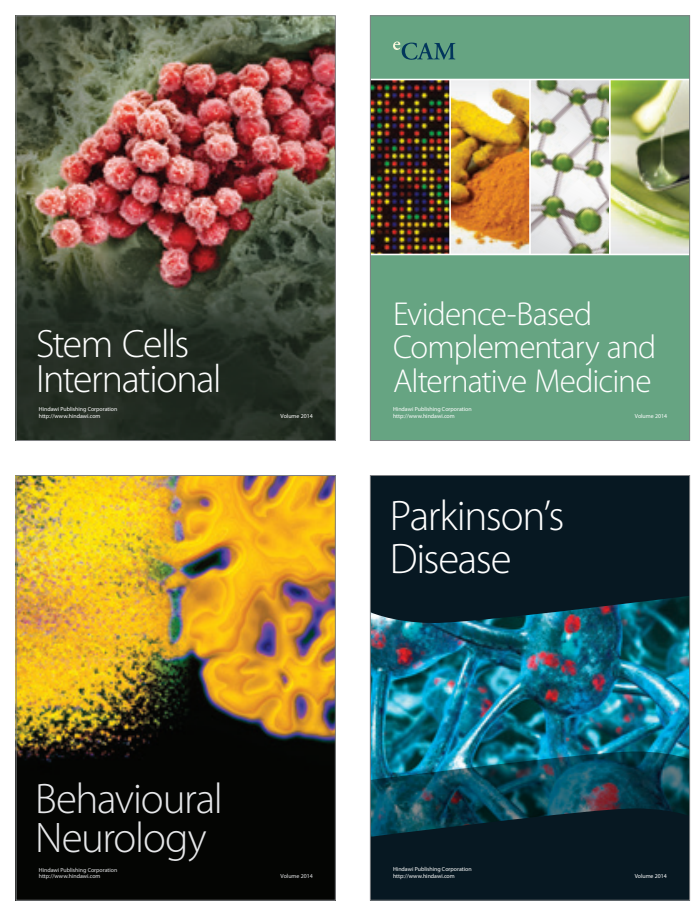

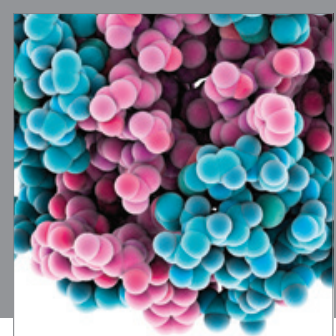

Journal of
Diabetes Research

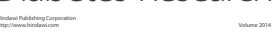

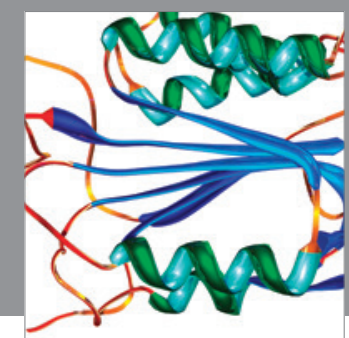

Disease Markers
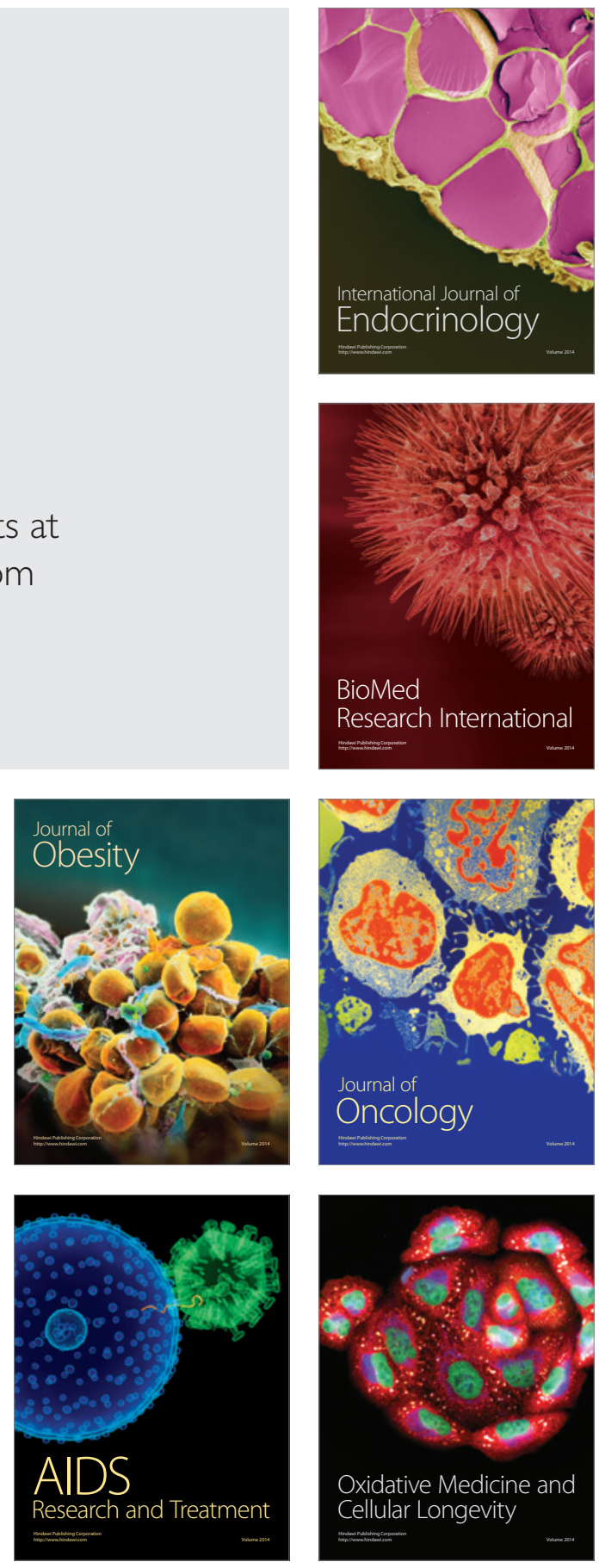